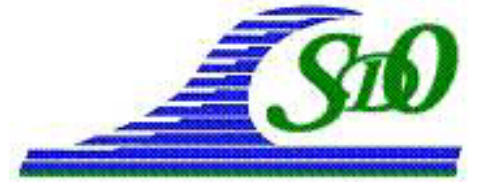

XI ${ }^{\text {èmes }}$ Journées Nationales Génie Côtier-Génie Civil

Les Sables d'Olonne, 22-25 juin 2010

DOI:10.5150/jngcgc.2010.053-D C Editions Paralia CFL

disponible en ligne - http://www.paralia.fr - available online

\title{
Analyse critique de l'utilisation des techniques de mesure topographique vidéo comme outil de gestion des plages sableuses
}

\author{
Damien DAILLOUX ${ }^{1}$, Didier RIHOUEY ${ }^{1}$, \\ Jérémy DUGOR ${ }^{1}$, Bruno CASTELLE ${ }^{2}$
}

\author{
1. CASAGEC-ADERA, UFR Sc. Et Tech., 1 Allée du Parc Montaury, \\ 64600 Anglet, France. \\ damien.dailloux@univ-pau.fr
}

2. Université de Bordeaux 1, UMR 5805 EPOC - OASU, 33405 Talence, France.

\section{Résumé :}

Depuis quelques années, l'utilisation de systèmes vidéo numériques pour quantifier les évolutions morpho-sédimentaires constitue une alternative autonome et peu coûteuse aux méthodes traditionnelles telles que la topographie. Le principe de la vidéo quantification appliquée au suivi des environnements côtiers est basé sur une technique de photogrammétrie qui permet de transformer une "image oblique brute" en une "image métrique plane". Depuis 2006, une succession de stations vidéo développées par CASAGEC ont été installées sur la côte Basque. Ainsi, environ $10 \mathrm{~km}$ de linéaire côtier sont quotidiennement surveillés par un ensemble de 6 stations vidéo. Cette surveillance vidéo quotidienne est accompagnée d'un suivi topographique régulier permettant d'évaluer la précision des mesures vidéo.

Nous proposons ici de réaliser une analyse critique de l'utilisation de la mesure topographique vidéo en tant qu'outil de gestion d'une plage sableuse. La comparaison des données vidéo et d'un grand nombre de mesures de terrain permet de mettre en avant les avantages et les limitations de la technique. L'automatisation de la détection $\mathrm{du}$ positionnement de la ligne d'eau et l'implémentation d'une valeur fiable de l'élévation du plan d'eau (marée, set-up, swash), sont autant de paramètres qui influencent la qualité de la mesure vidéo.

Les résultats obtenus montrent que les méthodes mises en œuvre sur la côte basque permettent d'obtenir une précision comparable à l'état de l'art. Cependant, cette précision ne permet pas de considérer la mesure topographique vidéo comme un outil fiable de gestion des plages sableuses si elle n'est pas complétée par d'autres approches. Mots-clés :

Suivi vidéo - Topographie - Ligne d'eau - Marée - Set-up - Swash - Outil de gestion 


\section{Introduction}

La mise en place d'une gestion durable et pérenne de la problématique d'érosion nécessite d'appréhender toutes les échelles spatio-temporelles d'évolution morphosédimentaire. Situé au fond du Golfe de Gascogne, le littoral Basque est soumis à des conditions moyennes de vagues de secteur ouest - nord ouest, de période $10 \mathrm{~s}$ et de hauteur significative $2 \mathrm{~m}$ (AUGRIS et al., 2009), et peut subir l'assaut de très fortes tempêtes (houle décennale : $H_{10}=11.5 \mathrm{~m}$ ). La marée, de type méso tidale semi-diurne (amplitude moyenne de $3.85 \mathrm{~m}$ en vive-eau et $1.85 \mathrm{~m}$ en morte-eau) engendre des circulations de faible intensité. Dans la zone de surf, les courants de marée sont négligeables devant ceux induits par le déferlement des vagues. Les caractéristiques spécifiques des plages Basques (plages de poche, enrochements, ruptures de pente) associées à une morphodynamique complexe et multi-échelles nécessitent la mise en place d'un dispositif de suivi le plus exhaustif possible.

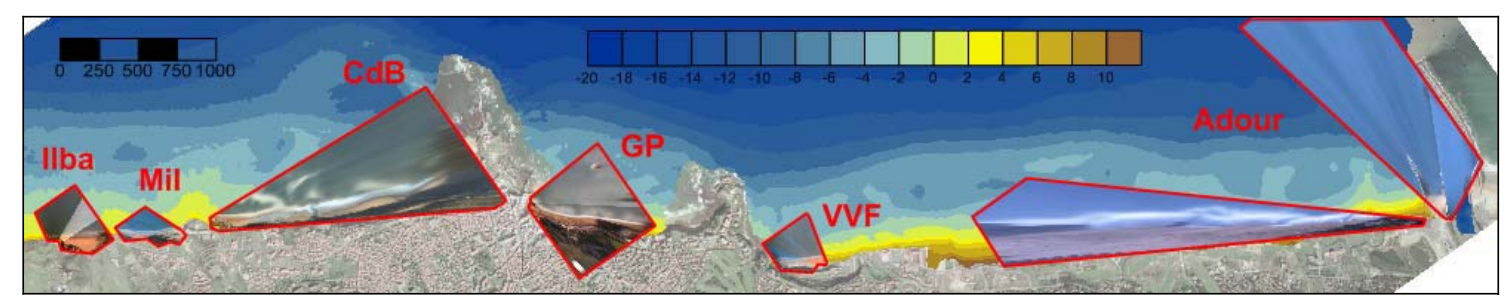

Figure 1. Représentation des 6 stations vidéo installées sur la côte Basque du Nord au Sud: l'Adour (Adour), le VVF (VVF), la Grande Plage (GP), la Côte des Basques $(\mathrm{CdB})$, la Milady (Mil), et Ilbarritz (Ilba).

Depuis quelques années, l'utilisation de systèmes vidéo numériques pour quantifier ces évolutions constitue une alternative autonome et peu coûteuse (SPECIAL ISSUE COASTAL ENGINEERING, 2007) aux méthodes traditionnelles telles que la topographie (PLANT \& HOLMAN, 1997 ; AARNINKHOFF et al., 2003). CASAGEC à développé un système de suivi vidéo permettant d'obtenir des données synchronisées à fréquence variable (jusqu'à $2 \mathrm{~Hz}$ ). Une station vidéo est composée de plusieurs caméras, disposées à proximité de la côte sur un point élevé. Elle permet d'acquérir des images instantanées (snap shot), et des images exposées sur 10 minutes (timex). Ce système est basé sur une technique de photogrammétrie (RIHOUEY et al., 2008) qui permet de transformer une "image oblique brute" en une "image métrique plane". Cette transformation repose sur une relation entre les coordonnées pixel $(\mathrm{u}, \mathrm{v})$ de l'image et la position $(\mathrm{x}, \mathrm{y}, \mathrm{z})$ correspondante dans le monde réel.

Depuis 2006, une succession de stations vidéo développées par CASAGEC ont été installées sur la côte Basque (http://kostasystem.univ-pau.fr/). Ainsi, environ $10 \mathrm{~km}$ de linéaire côtier sont quotidiennement surveillés par un ensemble de 6 stations vidéo (figure 1). 
Les données issues de ces stations sont ici confrontées à une base de données topographique DGPS pour réaliser une analyse critique de la topographie vidéo en tant qu'outil de gestion d'une plage sableuse. Premièrement, la technique de mesure vidéo de la topographie intertidale est présentée. Une analyse comparative de 42 topographies vidéo et DGPS est ensuite réalisée dans la section 3. La section 4 propose une discussion sur la sensibilité de la méthode. Enfin, une analyse consacrée à l'évaluation du système vidéo en tant qu'outil d'aide à la gestion d'une plage sableuse conclut cet article.

\section{Mesure vidéo de la topographie intertidale}

Le principe de la mesure topographique vidéo est basé sur deux modèles distincts: (i) un modèle de détection horaire des positions $(x, y)$ de la ligne d'eau au cours d'un demi cycle de marée, (ii) et un modèle de calcul de l'élévation $(z)$ de ces lignes d'eau. L'ensemble des coordonnées $(x, y, z)$ est ensuite interpolé pour construire un modèle numérique de terrain de l'estran.

\subsection{Détection de la ligne d'eau}

Historiquement, les méthodes de détection vidéo de la ligne d'eau étaient basées sur des images en dégradé de gris et définissaient la ligne d'eau comme le maximum d'intensité provoqué par le déferlement des vagues au bord (PLANT \& HOLMAN, 1997). Avec l'apparition des caméras couleurs, les algorithmes de détection se sont basés sur la divergence de comportement des différentes bandes de couleurs entre l'eau et la plage. Certaines méthodes sont basées sur une approche optique souvent dépendantes du site d'étude (AARNINKOFF, 2003 ; OSORIO, 2005 ; ALMAR, 2009), alors que d'autres sont basées sur une approche statistique (MORICHON et al., 2006; DAILLOUX, 2008; RIHOUEY et al., 2008). Cette étude est réalisée à partir d'une technique statistique de segmentation basée sur la méthode des k-moyennes (DAILLOUX, 2008 ; RIHOUEY et al., 2008 ; RIHOUEY et al., 2009).

\subsection{Modèle d'élévation de la ligne d'eau}

Dans un environnement tidal, l'élévation de la ligne d'eau $\left(Z_{l}\right)$ peut être estimée par (AARNINKHOFF, 2003) :

$$
Z_{l}=Z_{0}+\eta_{m}+K_{S W} \frac{\eta_{S W}}{2}+\eta_{p}+\eta_{v}
$$

où $Z_{0}$ est le niveau de marée (SHOM), $\eta_{m}$ est l'élévation moyenne de la surface libre engendrée par le déferlement des vagues (set-up), $\eta_{S W}$ est le niveau maximum du jet de rive (swash), $K_{s w}$ une constante empirique introduite pour prendre en compte le décalage entre la ligne d'eau localisée par le système vidéo et l'oscillation transversale maximale du swash sur les 10 minutes d'acquisition de l'image timex, $\eta_{p}$ l'effet barométrique inverse (pression atmosphérique), et $\eta_{v}$ l'effet du vent. Des mesures ont 
montrées que pour un vent de $20 \mathrm{~m} / \mathrm{s}, \quad \eta_{v}$ n'était seulement que de $0.06 \mathrm{~m}$ (AARNINKOF, 2003). En revanche, les changements de pression atmosphérique induisent des variations du niveau d'eau de l'ordre de $+0.01 \mathrm{~m}$ pour une chute de $1 \mathrm{hPa}$. Toutefois, étant donné que la méthode vidéo est principalement appliquée lors de conditions calmes, l'action du vent et de la pression atmosphérique seront négligées en première approximation.

2.3 Déformation moyenne de la surface libre engendrée par le déferlement des vagues

Par soucis de simplicité de la méthode utilisée, une approche paramétrique a été préférée à une approche numérique. La formulation de HOLMAN et SALLENGER (1985), paramétrée sur des plages naturelles, a été retenue pour réaliser cette étude. Elle permet de prendre en compte les conditions de déferlement sur les valeurs du set-up et s'exprime par :

$\eta_{m}=0.45 * \xi_{0} * H_{0}$

- $H_{0}$ est la hauteur significative des vagues en eau profonde.

- $\quad \xi_{0}$ est le nombre d'Irribaren (BATTJES, 1974). Ce dernier est couramment utilisé pour différencier les conditions de déferlement. Sa formulation est donnée par l'équation suivante :

$\xi_{0}=\frac{m}{\left(H_{0} / L_{0}\right)^{0.5}}$

où $m$ est la pente de la partie intertidale, et $L_{0}$ est la longueur d'onde des vagues en eau profonde. Par souci de respect du caractère autonome de la vidéo, la pente de la plage intertidale est calculée à partir des topographies vidéo réalisées avec un modèle d'élévation simplifié $\left(Z_{l}=Z_{0}\right)$.

\section{4 $\underline{\text { Swash }}$}

Les études réalisées sur le jet de rive (swash) séparent les contributions des oscillations hautes fréquences $(\ngtr>0.05 \mathrm{~Hz})$ et de basses fréquences $(f<0.05 \mathrm{~Hz})$ sur la hauteur totale du jet de rive. L'élévation du jet de rive est donnée par :

$\eta_{S W}=\sqrt{R_{i g}^{2}+R_{s s}^{2}}$

où $R_{s s}$ est la hauteur du swash à haute fréquence :

$\frac{R_{s s}}{H_{0}}=0.69 * \xi_{0}-0.19$

pour des valeurs du nombre d'Irribaren supérieure à 0.275 , et $R_{s s}=0$ pour des valeurs du nombre d'Irribaren inférieures à 0.275 (RUESSINK et al., 1998 ; AARNINKHOFF et al., 2003); et où $R_{i g}$ est la hauteur de jet de rive à basse fréquence exprimée par (RUESSINK et al., 1998) :

$R_{\text {ig }} / H_{0}=0.65 * \tanh \left(3.38 * \xi_{0}\right)$ 


\section{XI $I^{\text {èmes }}$ Journées Nationales Génie Côtier - Génie Civil}

Les Sables d'Olonne, 22-25 juin 2010

\section{Validité de la méthode de topographie vidéo}

Afin d'évaluer la précision de la technique, une série de 42 topographies (réalisée via un DGPS centimétrique) mise en œuvre entre avril 2008 et décembre 2009, est comparée aux topographies vidéo. L'ensemble des relevés de terrain a été réalisé pour de faibles conditions d'agitation $\left(H_{s}<2 \mathrm{~m}\right)$ et pour des coefficients de marée compris entre 70 et 110. Les données du modèle Wavewatch III, qui donnent une bonne représentation de l'agitation au large de la côte Basque (ABADIE et al., 2005), sont utilisées en entrée des modèles de set-up et de swash. Les caractéristiques des stations vidéo et des plages étudiées sont présentées dans le tableau 1. Les principaux résultats obtenus sont présentés dans le tableau 2 .

Tableau 1. Caractéristiques des stations vidéo: le nombre de caméra (\# Cam), les dimensions de la zone couverte (Couv.), et la résolution transversale (RT) et longitudinale (RL) des pixels présents dans cette zone. Caractéristiques des plages étudiées: le type de plage (Ouverte, Poche), la présence de rupture de pente (Oui ou Non).

\begin{tabular}{lllllll}
\hline & \multicolumn{2}{l}{ Station vidéo } & \multicolumn{3}{c}{ Plage } \\
\cline { 2 - 7 } & \#Cam & Couv. $(\boldsymbol{m})$ & $\boldsymbol{R} \boldsymbol{T}$ & $\boldsymbol{R L}$ & Type & Rup \\
\hline Adour & 1 & $1500 * 200$ & $<1 m$ & $<30 m$ & $O$ & Oui \\
$\boldsymbol{V} \boldsymbol{V} \boldsymbol{F}$ & 3 & $400 * 400$ & $<1 m$ & $<2 m$ & O/P & Oui \\
$\boldsymbol{G P}$ & 4 & $500 * 500$ & $<1 m$ & $<5 m$ & $P$ & Oui \\
$\boldsymbol{C d} \boldsymbol{B} \boldsymbol{M}$ & 2 & $1800 * 400$ & $<1 m$ & $<15 m$ & $O$ & Non \\
Mil & 2 & $250 * 250$ & $<1 m$ & $<2 m$ & $P$ & Oui \\
$\boldsymbol{I l b a}$ & 3 & $400 * 400$ & $<1 m$ & $<1 m$ & $P / O$ & Oui \\
\hline
\end{tabular}

Tableau 2. Validation Vidéo / DGPS : nombre de topographies disponibles (\# Data), erreur quadratique moyenne par station (ERMS), pente moyenne estimée par la vidéo ( $m V i d)$ et le DGPS (mDGPS), et leurs déviations standards respectives (DS mVid, DS $m D G P S)$.

\begin{tabular}{lllllll}
\hline & \# Data & ERMS & mVid & DS mVid & mDGPS & DS mDGPS \\
\hline Adour & 2 & 0.67 & 8.2 & 2.4 & 9.3 & 0.48 \\
$\boldsymbol{V} \boldsymbol{V F}$ & 6 & 0.91 & 9.67 & 1.81 & 9.9 & 1.33 \\
$\boldsymbol{G P}$ & 13 & 0.5 & 6.7 & 1.69 & 8.5 & 1.49 \\
$\boldsymbol{C d B}$ & 3 & 0.29 & 2.5 & 1.2 & 2.7 & 0.7 \\
$\boldsymbol{M i l}$ & 6 & 0.52 & 8.8 & 3.7 & 9.7 & 2.9 \\
$\boldsymbol{I l b a}$ & 15 & 0.46 & 6.2 & 2.59 & 8.1 & 2.01 \\
\hline
\end{tabular}

Il existe une très bonne corrélation sur l'ensemble des topographies DGPS et vidéo sur l'ensemble des 5 plages $\left(R_{\text {moy }}^{2}=0.97\right)$. La technique permet donc de bien représenter la 
morphologie globale des plages étudiées. Cependant, l'erreur quadratique moyenne est très variable pour la plupart des stations.

L'influence de la pente de la plage semble être déterminante dans la validité de la méthode. Les meilleurs résultats $(E R M S=0.29$, uniquement à parti de trois mesures) sont obtenus pour la plage de la Côte des Basques où la pente est la plus faible (2.5\%), alors que des erreurs comprises entre $0.6 \mathrm{~m}$ et $1 \mathrm{~m}$ sont enregistrées pour les plages à forte pente (Adour et VVF). Cependant, il n'existe encore que trois mesures réalisé sur la côte des Basque. De plus, l'hypothèse d'une plage plane ne permet pas de prendre en compte les morphologies irrégulières de certaines plages, biaisant ainsi les résultats obtenus. En effet, la présence quasi quotidienne de la rupture de pente de la grande plage de Biarritz augmente l'erreur globale de l'estimation autour d'une valeur stable de $0.5 \mathrm{~m}$. Au contraire, les plages de la Milady et d'Ilbarritz ont une morphologie beaucoup plus variable dans le temps $(D S m V i d>2.5)$ que les autres plages. Alors que l'erreur peut être faible $(<0.3 \mathrm{~m})$ lorsque les plages présentent une pente uniforme, elle peut drastiquement augmenter lors de présence d'une rupture de plage (ERMS $>0.6$ ).

De manière générale, il est important de noter que l'implémentation du modèle paramétré de l'élévation de la ligne d'eau a permis d'améliorer la précision de la technique par rapport aux résultats obtenus par RIHOUEY et al. (2008). A l'exception des sites de l'Adour et du VVF, l'erreur, inférieure à environ $0.5 \mathrm{~m}$, est comparable à l'état de l'art. Les études réalisées à partir des méthodes SLIM (PLANT \& HOLMAN, 1997) et IBM (AARNINKHOFF et al., 2003) sur les plages de Duck (NC), Teignmouth (UK) et Egmond (NL), montrent des erreurs comprises entre $0.14 \mathrm{~m}$ et $0.67 \mathrm{~m}$. De plus, il est important de souligner qu'il existe des différences morphodynamiques très importantes entre les plages de Biarritz et les plages de Duck et d'Egmond. En effet, les plages de Biarritz sont plus pentues et soumises à des conditions de houles plus importantes.

\section{Discussion}

Si les équations paramétrées utilisées dans cette étude peuvent être considérées comme fiables, une analyse de sensibilité de la méthode par rapport aux entrées des modèles est nécessaire. Les deux principales sources d'erreurs sont l'estimation du niveau de marée et le calcul de la pente de la plage intertidale.

\subsection{Influence du niveau de marée}

Les données de marée du SHOM à Saint Jean de Luz ont été comparées aux données de marée SONEL du marégraphe présent dans la baie, sur une période de deux ans (2008 et 2009). Alors que les corrélations entre les deux sets de données sont excellentes $\left(R^{2}>0.99\right)$, le biais montre des valeurs importantes pouvant atteindre des valeurs maximales de plus de $50 \mathrm{~cm}$ dues aux perturbations atmosphériques et aux conditions de vent. Cependant, l'erreur est inférieure à $20 \mathrm{~cm}$ au cours des jours de mesures où le 
niveau d'agitation est faible. Elle n'explique donc pas à elle seule les erreurs quadratiques moyennes pouvant atteindre des valeurs périodiques de $1 \mathrm{~m}$.

\subsection{Influence de la pente de la plage intertidale}

Les pentes obtenues par la vidéo sous-estiment constamment celles obtenues au DGPS (Tableau 2). De plus, les valeurs de pente obtenues sont des valeurs moyennées sur l'ensemble de la surface de chacune des topographies. Ainsi, la présence de rupture de plage, ou de banc de déferlement biaise les résultats. Les valeurs de set-up et de swash étant calculées à partir de cette pente moyenne, leurs variations longitudinales et transversales ne sont pas prises en compte. Ainsi, lorsque la pente de la plage intertidale est uniforme, la formulation de set-up et du swash semble appropriée. Par contre, lorsque la plage intertidale possède une morphologie irrégulière, les valeurs de set-up et de swash sont surestimées dans la partie basse de la plage (terrasse de basse mer) et sous-estimées dans la partie haute de la plage (rupture de plage).

\section{Le système vidéo en tant qu'outil d'aide à la gestion}

Aux vues des résultats obtenus par la mesure topographique vidéo de plage à morphologies complexes et/ou irrégulières (forte pente, rupture de pente, barre de déferlement), cette technique ne semble pas être adéquate pour dresser un budget sédimentaire complet et précis. En effet, la précision de la mesure peut engendrer des erreurs d'estimations de volume sédimentaire de plusieurs dizaines (Milady et Ilbarritz) à plusieurs centaines (Adour, VVF, GP, $\mathrm{CdB}$ ) de millier de $\mathrm{m}^{3}$. L'influence du calcul de la pente locale et de l'estimation du niveau d'eau (marée, set-up et swash) sont autant de paramètres influant sur la fiabilité de la méthode. Néanmoins, la méthode utilisée dans cette étude donne des résultats semblables aux différentes techniques utilisées à travers le monde (ERMS $=0.3$ à $0.7 \mathrm{~m}$, PLANT et al., 2007), pour des morphologies pourtant plus complexes que celles précédemment testées.

Cependant le système vidéo permet d'obtenir d'autres types d'informations nécessaires à une gestion pérenne des problématiques d'érosion (SPECIAL ISSUE COASTAL ENGINEERING, 2007). L'identification d'Indicateur d'Etat Côtier (IEA) permet d'apporter différentes applications au système vidéo tel que :

- Le suivi mensuel à quotidien de la ligne d'eau des environnements micro tidaux, qui permet d'établir un bilan complet de la réponse de la plage suite à un rechargement sableux ou à l'implantation d'une protection côtière (RIHOUEY et al., 2009);

- La localisation de la ligne d'eau lors des surcotes engendrées par les plus fortes tempêtes, qui permet de définir le Domaine Publique Maritime (DPM) ;

- la position quotidienne de la ligne d'eau pour un niveau d'eau constant dans les environnements tidaux, qui permet d'obtenir des modèles conceptuels d'évolution morphodynamique de la plage. 
Thème 3 - Instrumentation, mesures, imagerie et télédétection

\section{Références bibliographiques}

AARNINKHOF S.G.J., TURNER I.L., DRONKERS T.D.T., CALJOUW M., NIPIUS L. (2003). A video-based technique for mapping intertidale beach bathymetry. Coastal Engineering, 49, pp 275-289. doi:10.1016/S0378-3839(03)00064-4

ABADIE S., BUTEL R., DUPUIS H., BRIERE C. (2005). Paramètres statistiques de la houle au large de la côte sud-aquitaine. Comptes Rendus Geosciences, 337, pp 769-776. doi:10.1016/j.crte.2005.03.012

ALMAR R. (2009). Morphodynamique littorale haute fréquence par image vidéo. Thèse de doctorat, Université de Bordeaux.

AUGRIS C., CAILL-MILLY N., DE CASAMAJOR M.N., (2009). Atlas thématique de l'environnement marin du Pays Basque et sud des Landes. Edition Quae, pp 17-22.

DAILLOUX D. (2008). Video measurements of the Adour plume dynamc and its surface water optical characteristics. Thèse de doctorat, Université de Pau et des Pays de l'Adour.

HOLMAN R.A., SALLENGER A.H. (1985). Setup and Swash on a Natural Beach. Journal of Geophysical Research, 90, pp 945-953. doi:10.1029/JC090iC01p00945

MORICHON D., DAILLOUX D., AARNINKHOF S., ABADIE S. (2007). Using a shore based video system to hourly monitor storm water plumes (Adour River, Bay of Biscay). Journal of Coastal Research, 24, pp 133-140. doi:10.2112/06-0705.1

OSORIO A. (2005). Video-derive techniques and methodologies for coastal management. Phd Thesis. Universidad de Cantabria.

PLANT N.G., AARNINKHOF S.G., TURNER I.L., KINGSTON K.S. (2007). The performance of shoreline detection models applied to video imagery, J. of Coastal Res., 23, pp 658-670. doi:10.2112/1551-5036(2007)23[658:TPOSDM]2.0.CO;2

PLANT N.G, HOLMAN R.A, (1997). Intertidal beach profile estimation using video image. Marine Geology 140, pp 1-24. doi:10.1016/S0025-3227(97)00019-4

RIHOUEY D., BLANGUY A., DUGOR J., DAILLOUX D. (2008). Technique vidéo appliquée à la topographie de la plage intertidale. $\mathrm{X}^{\mathrm{èmes}}$ Journées Nationales Génie Civil - Génie Côtier, Sophia Antipolis, pp 625-634. doi:10.5150/jngcgc.2008.060-R

RIHOUEY D., DUGOR J., DAILLOUX D., MORICHON D. (2009). Application of remote sensing video systems to coastal defence monitoring. Journal of Coastal Research, SI 56, pp 1582-158.

RUESSINK B.G., KLEINHANS M.G., VAN BEUKEL P.G.L., (1998). Observations of swash under highly dissipative conditions. Journal of Geophysical Research 103, pp 3111-3118. doi:10.1029/97JC02791

SPECIAL ISSUE COASTAL ENGINEERING (2007). The CoastView Project: Developing coastal video monitoring systems in support of coastal zone management, Coastal Engineering, 54, Special issue edited by M. Davidson and R. Medina, pp 461-576. 